\title{
Stability of the solar tachocline with magnetic fields
}

\author{
R. Arlt ${ }^{1, \star}$, A. Sule ${ }^{2}$, and R. Filter ${ }^{1}$ \\ 1 Astrophysikalisches Institut Potsdam, An der Sternwarte 16, D-14482 Potsdam, Germany \\ 2 Homi Bhabha Centre for Science Education, Mankhurd, Mumbai, 400088, India
}

Received 2007 Sep 7, accepted 2007 Nov 12

Published online 2007 Dec 15

Key words Sun: magnetic fields - instabilities

The stability of magnetic fields in the solar tachocline is investigated. We present stability limits for higher azimuthal wave numbers and results on the dependence of the stability on the location of toroidal magnetic fields in latitude. While the dependence of the wave number with the largest growth rate on the magnetic field strength and the magnetic Prandtl number is small, the dependence on the magnetic Reynolds number Rm indicates that lowest azimuthal modes are excited for very high $\mathrm{Rm}$. Upon varying the latitudinal position of the magnetic field belts, we find slightly lower stability limits for high latitudes, and very large stability limits at latitudes below $10^{\circ}$, with little dependence on latitude in between. An increase of the maximum possible field was achieved by adding a poloidal field. The upper limit for the toroidal field which can be stored in the radiative tachocline is then $1000 \mathrm{G}$, compared to about $100 \mathrm{G}$ for a purely toroidal field as was found in an earlier work.

\section{Introduction}

The paper is concerned with the magnetic stability of the solar tachocline, which is the transition zone between the convection zone with chiefly latitudinal gradients in angular velocity and the radiative core with nearly uniform rotation. The thickness is roughly $4 \%$ of the solar radius. Helioseismology has confirmed the strong radial shear within the tachocline and the fairly small radial extent of that zone (e.g. Basu \& Antia 2001).

A plausible formation mechanism for the tachocline of magnetic origin was proposed by Rüdiger \& Kitchatinov (1997) and Gough \& McIntyre (1998). The coupling of a core magnetic field with the differential rotation in the radiative interior below the convection zone leads to a thin transition layer, thereby causing toroidal magnetic fields of about $200 \mathrm{G}$. As a large class of solar dynamo models assume the tachocline to be the seat of the solar dynamo (e.g. Choudhuri \& Gilman 1987; D'Silva \& Choudhuri 1993), the toroidal field strength of the belts should be of the order of $10^{5} \mathrm{G}$ to give rise to the visible emergence of sunspots at the surface at low latitudes.

An early stability analysis of the toroidal magnetic fields in the solar case was done by Caligari et al. (1995) and was followed up by Ferriz-Mas \& Schüssler (1996). They used a one-dimensional flux tube approximation and concluded that the stability limit is in excess of $10^{4} \mathrm{G}$ in the tachocline as well as in the lower convection zone. In a different approach, nearly every toroidal magnetic field was found to be unstable at zero rotation by Vandakurov (1972) and Tayler (1973).

\footnotetext{
^ Corresponding author: rarlt@aip.de
}

A number of authors extended the 2-dimensional hydrodynamic formulation of Watson (1981) to include magnetic fields (e.g. Gilman \& Fox 1997; Gilman \& Dikpati 2000; Cally 2003; Cally et al. 2003; Dikpati et al. 2004). Most recently, Arlt et al. (2007) have studied the MHD stability of the lowest non-axisymmetric azimuthal mode in the solar tachocline, with a full tachocline rotation profile. An upper limit for the toroidal magnetic field of about $100 \mathrm{G}$ was found.

It has been speculated that the true stability limits can be identified only by studying higher azimuthal modes $(1<$ $m \lesssim 10)$. Gilman \& Dikpati (2000) have reported that comparatively higher azimuthal modes ( $m=4$ to 7 ) turn out to be the most unstable ones if the thin toroidal magnetic field belt is placed at mid-latitudes. The lowest azimuthal modes, however, dominate when the field belt is placed at very high or very low latitudes. Brun \& Zahn (2006), in their examination of the magnetic confinement of the solar tachocline, found the tachocline becoming unstable with a dominant $m=1$ mode close to the pole, but higher $m$ modes can be seen to be present at slightly lower latitudes, for a toroidal field build-up of roughly a few hundred Gauss.

In this Paper, we extend the analysis of Arlt et al. (2007) in various directions. We compute growth rates for higher azimuthal modes, show the dependence of the stability on the field belt position in latitude, and present results from combined toroidal and poloidal magnetic fields.

\section{Stability analysis model}

The numerical setup is that of a spherical shell, extending from the inner radius $R_{\mathrm{i}}=0.6$ to the outer radius 
$R_{\mathrm{O}}=0.7$. We are applying the spherical spectral MHD code by Hollerbach (2000) for the computations. The code solves the incompressible MHD equations in Boussinesq approximation; we reduced the numerics to the solution of the linearized induction and momentum equation for the stability analysis of nonaxisymmetric perturbations in the magnetic field, $\boldsymbol{b}$, and the velocity field, $\boldsymbol{u}$. The equations are

$$
\begin{aligned}
\frac{\partial \boldsymbol{u}}{\partial t}= & \operatorname{Rm}[\boldsymbol{u} \times \nabla \times \boldsymbol{U}+\boldsymbol{U} \times \nabla \times \boldsymbol{u}-\nabla(\boldsymbol{u} \cdot \boldsymbol{U})]+ \\
& S[(\nabla \times \boldsymbol{b}) \times \boldsymbol{B}+(\nabla \times \boldsymbol{B}) \times \boldsymbol{b}]- \\
& \nabla p+\operatorname{Pm} \triangle \boldsymbol{u}, \\
\frac{\partial \boldsymbol{b}}{\partial t}= & \nabla \times(\operatorname{Rm} \boldsymbol{U} \times \boldsymbol{b}+S \boldsymbol{u} \times \boldsymbol{B})-\triangle \boldsymbol{b} .
\end{aligned}
$$

The axisymmetric background rotation velocity profile $\boldsymbol{U}=(0,0, r \sin \theta \Omega)$ is decoupled from the nonaxisymmetric perturbations, as is the background magnetic field $\boldsymbol{B}$. The symbols $r, \theta$, and $\phi$ have the usual meanings of radius, colatitude, and longitude. Upon making the equations non-dimensional, the magnetic Reynolds number $\mathrm{Rm}=R_{\mathrm{o}}^{2} \Omega_{\mathrm{eq}} / \eta$, the Lundquist number $S=R_{\mathrm{o}} B_{0} / \sqrt{\mu \rho} \eta$, and the magnetic Prandtl number $\mathrm{Pm}=\nu / \eta$ appear, with $\nu$ and $\eta$ being the microscopic viscosity and magnetic diffusivity of the plasma, respectively, $\Omega_{\text {eq }}$ the equatorial angular velocity at the bottom of the convection zone, and $\mu$ and $\rho$ the magnetic permeability and the density, respectively. The rotation profile gives the axisymmetric background velocity

$\boldsymbol{U}=\left(0,0, r \sin \theta\left(1-\alpha_{2} \cos ^{2} \theta\right)\right)$,

where $\alpha_{2}$ describes the strength of the differential rotation.

The radial boundary conditions for the nonaxisymmetric flow are stress-free. The magnetic field faces vacuum conditions at both radial boundaries. The outer boundary is the bottom of the convection zone which provides very high magnetic diffusivity and is thus set to vacuum. The inner boundary has no physical meaning; we still set this to vacuum, because we do not expect toroidal magnetic fields to penetrate the solar core deeply (the condition sets $b_{\phi}$ to zero) and poloidal fields can extend into the core without building up currents in the physically non-existing boundary.

The code employs Chebyshev polynomials of degrees $k$ for the radial decomposition and spherical harmonics of degrees $(l, m)$ for the horizontal decomposition. The quantities which are decomposed spectrally are the potentials $e$, $f, g$, and $h$ forming $\boldsymbol{u}=\nabla \times\left(e \boldsymbol{e}_{r}\right)+\nabla \times \nabla \times\left(f \boldsymbol{e}_{r}\right)$ and $\boldsymbol{B}=\nabla \times\left(g \boldsymbol{e}_{r}\right)+\nabla \times \nabla \times\left(h \boldsymbol{e}_{r}\right)$ with $\boldsymbol{e}_{r}$ being the radial unit vector. The typical spectral truncation was at 40 Chebyshev polynomials and 80 Legendre polynomials, while individual Fourier modes were evolved in this linear analysis.

\section{Toroidal magnetic fields}

In Arlt et al. (2007), we reported that the solar tachocline may be unstable for a field of roughly $100 \mathrm{G}$. We extend

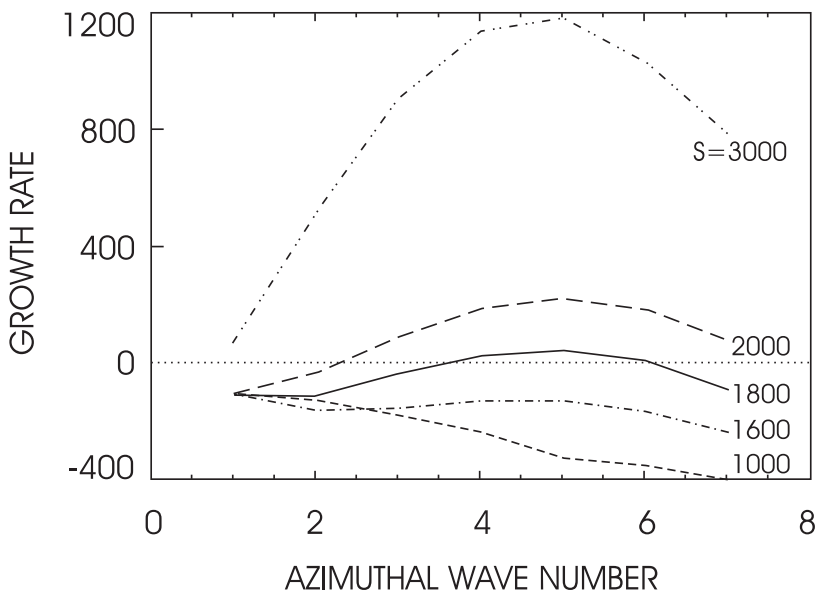

Fig. 1 Dependence of the stability of various azimuthal modes $m$ on the magnetic field strength in terms of the Lundquist number $(\mathrm{Rm}=10000, \mathrm{Pm}=1)$.

the linear computations to higher azimuthal modes and different locations of the magnetic field belt in latitude. The differential rotation $\left(\alpha_{2}\right)$ was fixed at $20 \%$. This value is a suitable choice for the upper boundary of the non-turbulent tachocline. The results do not actually change very much for small deviations of $\alpha_{2}$ from the chosen value, as was shown in Arlt et al. (2007).

The axisymmetric background magnetic field $\boldsymbol{B}=$ $\left(0,0, B_{\phi}\right)$ takes forms of

$B_{\phi}=B_{0} \sin ^{p} \theta \cos ^{q} \theta \sin ^{2}\left[\pi\left(\frac{r-R_{\mathrm{i}}}{R_{\mathrm{o}}-R_{\mathrm{i}}}\right)^{4}\right]$,

where $p$ and $q$ control the position of the maximum $B_{\phi}$ in latitude. For the following subsections, we set $p=2$ and $q=1$ resulting in a maximum $B_{\phi}$ at a latitude of $35^{\circ}$ from the equator. Since the investigated modes with $m>0$ entirely decouple from the axisymmetric state in the linearized system, it is not essential for their stability that the background setup is in equilibrium.

\subsection{Azimuthal modes with $m>1$}

Instead of searching for the stability limits of individual $m$ modes, the growth rates of the modes are computed and compared at given $S$ in order to save computation time. We suppose that the mode with the largest growth rate is also the one which becomes unstable first. The growth rate here is defined by the inverse e-folding time of the lowest latitudinal mode $(1, m)$.

Figure 1 shows the dependence of the growth rate on the azimuthal mode number $m$ for various Lundquist numbers corresponding to various background field strengths. The magnetic Reynolds number was fixed at $\mathrm{Rm}=10000$, the magnetic Prandtl number was $\mathrm{Pm}=1$. There is a maximum in the set of growth rates at $m=5$ for magnetic fields above $S \sim 1500$. There is nearly no dependence of the most easily excited mode on the Lundquist number, provided the growth rate is positive. 


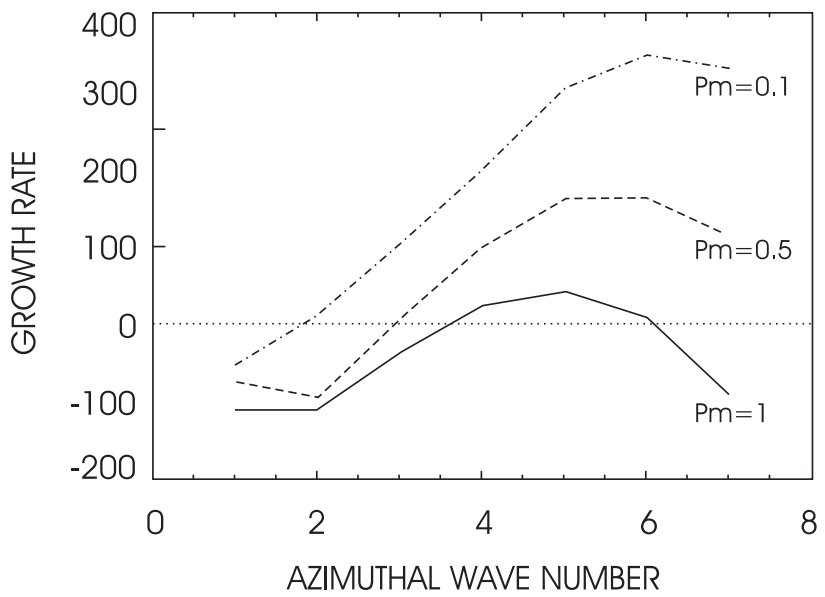

Fig. 2 Dependence of the stability of various azimuthal modes $m$ on the magnetic Prandtl number $(\mathrm{Rm}=10000, S=1800)$.

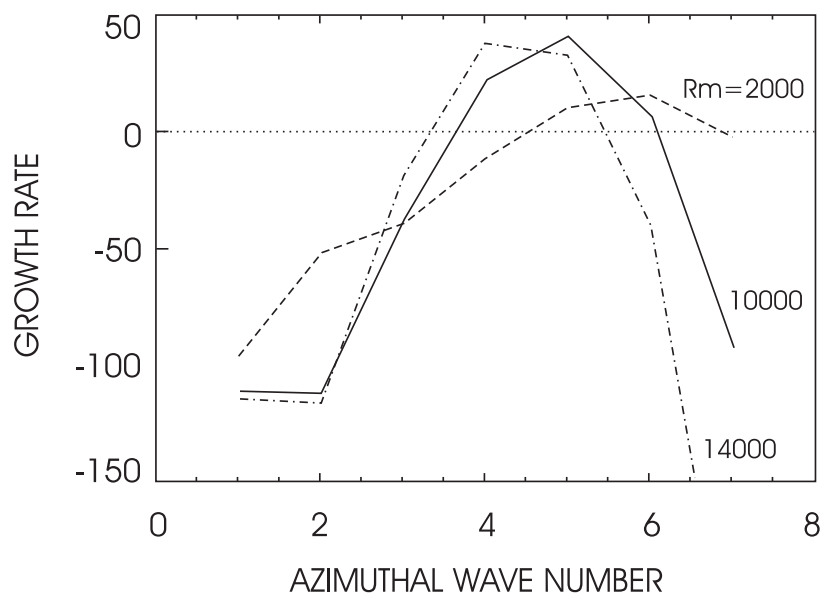

Fig. 3 Dependence of the stability of various azimuthal modes $m$ on the magnetic Reynolds number $(\mathrm{Pm}=1, S=1800)$.

The effect of the magnetic Prandtl number on the growth rate of $m=1-7$ is shown in Fig. 2. Again, the magnetic Reynolds number was fixed at $\mathrm{Rm}=10000$. While we found $m=5$ to be the most unstable mode for $\mathrm{Pm}=1$, a slight shift to $m=6$ is found for $\mathrm{Pm}=0.1$. The magnetic Prandtl number is another order of magnitude smaller in the Sun, and we expect the most easily excited mode to be one of $m=7$ or 8 , but definitely below $m=10$.

Finally, the most critical influence is that of the magnetic Reynolds number, since the $\mathrm{Rm}$ of the simulations is several orders of magnitudes below the value in the radiative interior of the Sun. Figure 3 shows again sets of growth rates versus azimuthal mode number $m$, this time for various $\mathrm{Rm}$. Pm was reset to unity. There is a trend towards lower $m$ as $\mathrm{Rm}$ is increased. Modes higher than $m=1$ make computations more difficult, and the full $m$-set could only be obtained up to $\mathrm{Rm}=14000$. A guess for the strong extrapolation to the very high solar $\operatorname{Rm}$ may favour $m=1$ for the most easily excited mode in the Sun. The tendency of exciting slightly higher $m$ for solar magnetic Prandtl numbers may not alter this result, but this is speculation.

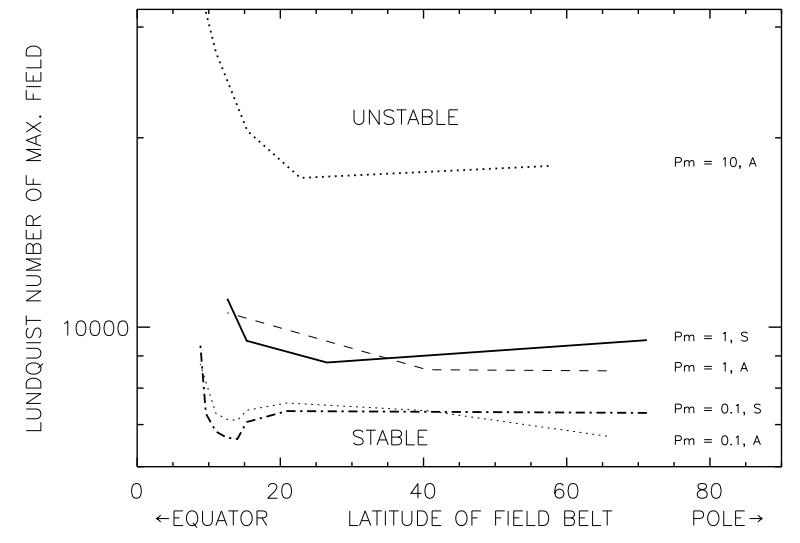

Fig. 4 Dependence of the stability on the position of the magnetic field belt in latitude. Stability lines for magnetic Prandtl numbers of $0.1,1$, and 10 are plotted. The symbols 'A' and ' $S$ ' refer to the stability of antisymmetric and symmetric flow perturbations, respectively. The fact that the critical Lundquist numbers for lower $\mathrm{Pm}$ are lower, does not mean the field in physical units is weaker, since the Lundquist number changes with $\eta^{-1}$ as does Pm.

\subsection{Latitude dependence}

The position of the maximum $B_{\phi}$ in the previous Section has always been at a latitude of $35^{\circ}$. We now vary the latitude of the magnetic field belts by varying $p$ and $q$ in (4). The toroidal background field thus faces negative radial $\Omega$ gradients at high latitudes and positive radial $\Omega$ gradients at low latitudes. The range was from $p=48$ and $q=1$ corresponding to a latitude of $80.8^{\circ}$ to $p=2, q=21$ corresponding to $16^{\circ}$ latitude, where $p$ takes only even values and $q$ takes only odd values. Note that we added an amplitude correction to (4) so that the maximum of $B_{\phi}$ is always \pm 1 . Only the $m=1$ mode is tested for instability here.

Figure 4 shows the dependence of the stability limits in terms of Lundquist numbers versus the belt latitude. Sets for different configurations are shown; ' $A$ ' refers to the stability of an antisymmetric flow, i.e. $u_{r}(\theta)=-u_{r}(\pi-\theta), u_{\theta}(\theta)=$ $u_{\theta}(\pi-\theta), u_{\phi}(\theta)=-u_{\phi}(\pi-\theta)$; 'S' refers to a symmetric perturbation, i.e. $u_{r}(\theta)=u_{r}(\pi-\theta), u_{\theta}(\theta)=-u_{\theta}(\pi-\theta)$, $u_{\phi}(\theta)=u_{\phi}(\pi-\theta)$. We also varied the magnetic Prandtl number from $\mathrm{Pm}=0.1$ to 10 .

All the sets show relatively little dependence on the latitude between $30^{\circ}$ and $60^{\circ}$ latitude. There is strongly increased stability only at latitudes below latitudes of $10^{\circ}$. This is in line with the very small growth rates found by Dikpati et al. (2003) at latitudes below $\sim 15^{\circ}$ as is shown in their Figure 11. We also find that the antisymmetric flows are more easily excited than symmetric flows at high latitudes, i.e. above $\sim 40^{\circ}$. This again agrees with the findings of Dikpati et al. (2003) who encountered significant growth rates first in the antisymmetric $m=1$ mode upon increasing the magnetic field strength (they used a different symmetry definition and actually referred to that mode as being the symmetric one). 


\section{Toroidal combined with poloidal fields}

The real environment in the solar tachocline will also involve weak poloidal magnetic fields. This Section deals with the change in stability limits for toroidal fields if they are combined with a weak poloidal component.

In order to produce a consistent background magnetic field with poloidal and toroidal components, we solve the induction equation under the influence of a differential rotation which has the simplified form $\Omega=\Omega_{\mathrm{eq}}\left(1-\alpha_{2} \cos ^{2} \theta\right)$. A background poloidal magnetic field of a shape suggested by Rüdiger \& Kitchatinov (1997) is subject to differential rotation and provides us with a suitable toroidal magnetic field. The axisymmetric induction equation for generating the $B_{\phi}$ field is

$\frac{\partial \boldsymbol{B}}{\partial t}=\nabla \times\left(\boldsymbol{U} \times \boldsymbol{B}+\boldsymbol{U} \times \boldsymbol{B}_{0}\right)+\nabla^{2} \boldsymbol{B}$,

where

$\boldsymbol{B}_{0}=\left(2 \cos \theta\left[1-\frac{r}{R_{\mathrm{o}}}\right], \sin \theta\left[\frac{3 r}{R_{\mathrm{o}}}-2\right], 0\right)$.

The poloidal background field does not penetrate the outer boundary, i.e. the boundary with the convection zone, as was suggested by Rüdiger et al. (2005). The problem reduces to solving the $m=0$ mode with even $l$ in the potential $g$ (antisymmetric magnetic field). The solution is steady-state, and we keep $B_{\phi}$ and $\nabla \times\left(B_{\phi} \boldsymbol{e}_{\phi}\right)$ for the stability analysis. The resulting $B_{\phi}$ does not exceed the poloidal field as much as in the Sun, since we do not reach sufficiently high magnetic Reynolds numbers. For that reason, we combine the resulting $B_{\phi}$ and its curl with the original poloidal magnetic field at an arbitrary ratio for the stability analysis which is done employing Eqs. (2) and (1).

A ratio of $B_{\phi} / B_{\text {pol }}=100$ was adopted for the stability analysis. The dependence of the critical Lundquist number on $\mathrm{Rm}$ is shown in Fig. 5 for $\mathrm{Pm}=1$. Solar magnetic Reynolds numbers in a radiative environment are much higher than the maximum of 100000 used here. We have to extrapolate the Lundquist numbers to solar Rm in the same way as was done by Arlt et al. (2007). The combination of a poloidal magnetic field with a consistently generated, 100 times stronger toroidal magnetic field yields a stability limit of $980 \mathrm{G}$ for the tachocline.

\section{Conclusions}

The stability analysis of Arlt et al. (2007) led to a stability limit of about $100 \mathrm{G}$ for purely toroidal magnetic fields in the solar tachocline and $m=1$ perturbations. Here we extended the analysis to higher azimuthal modes and find that in the radiative tachocline environment, very low $m$ will be excited, with $m=1$ probably being the most easily excitable. We also change the latitudinal position of the

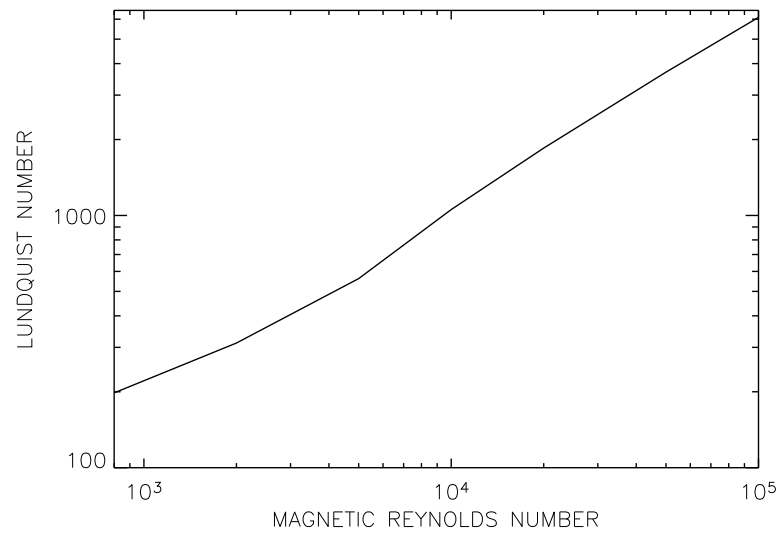

Fig. 5 Stability limit of a combination of toroidal and poloidal magnetic field versus magnetic Reynolds number Rm. The magnetic Prandtl number Pm was unity.

magnetic field belt. Slightly lower stability limits for high latitudes was found, as well as very (numerically unreachably) high stability limits for latitudes below $10^{\circ}$, with little dependence on latitude in between. The combination of the toroidal fields with a poloidal field which is 100 times weaker delivered a stabilizing effect, and the upper limit for field storage in the tachocline is then near $1000 \mathrm{G}$. This can be considered an upper limit, since the poloidal magnetic field is supposed to be weaker in the tachocline than $B_{\phi} / 100$ (much less than $1 \mathrm{G}$, Rüdiger et al. 2005). Our conclusion from Arlt et al. (2007) remains valid, that toroidal magnetic fields of $10^{4}-10^{5} \mathrm{G}$ cannot be stored in the solar tachocline.

Acknowledgements. AS thanks the Deutsche Forschungsgemeinschaft for their support by grant No. Ru 488/15-1. RF thanks the Astrophysikalisches Institut Potsdam for the hospitality.

\section{References}

Arlt, R., Sule, A., Rüdiger, G.: 2007, A\&A 461, 295

Basu, S., Antia, H.M.: 2001, MNRAS 324, 498

Brun, A.S., Zahn, J.-P.: 2006, A\&A 457, 665

Caligari, P., Moreno-Insertis, F., Schüssler, M.: 1995, ApJ 441, 886

Cally, P.S.: 2003, MNRAS 339, 957

Cally, P.S., Dikpati, M., Gilman, P.A.: 2003, ApJ 582, 1190

Choudhuri, A.R., Gilman, P.A.: 1987, ApJ 316, 788

D'Silva, S., Choudhuri, A.R.: 1993, A\&A 272, 621

Dikpati, M., Cally, P., Gilman, P.A.: 2004, ApJ 610, 597

Dikpati, M., Gilman, P.A., Rempel, M.: 2003, ApJ 596, 680

Ferriz-Mas, A., Schüssler, M.: 1996, GApFD 81, 233

Gilman, P.A., Dikpati, M.: 2000, ApJ 576, 1031

Gilman, P.A., Fox, P.: 1997, ApJ 484, 439

Gough, D.O., McIntyre, M.E.: 1998, Nature 394, 755

Hollerbach, R.: 2000, IJNMF 32, 773

Rüdiger, G., Kitchatinov, L.L.: 1997, AN 318, 273

Rüdiger, G., Kitchatinov, L.L., Arlt, R.: 2005, A\&A 444, L53

Tayler, R.J.: 1973, MNRAS 161, 365

Vandakurov, Yu.V.: 1972, SvA 16, 265

Watson, M.: 1981, GApFD 16, 285 